\title{
On the emerging role of neuroimaging in determining functional and structural brain integrity induced by physical exercise: impact for predictive, preventive, and personalized medicine
}

\author{
Henning Boecker
}

Received: 10 May 2011 / Accepted: 12 June 2011 /Published online: 8 July 2011

(C) European Association for Predictive, Preventive and Personalised Medicine 2011

\begin{abstract}
Physical exercise contributes to maintenance of physical health. There is growing evidence, both from animal work and from human epidemiological and longitudinal intervention studies, that physical exercise also impacts directly on the brain, mediating structural integrity and improving cognitive functioning. Modern neuroimaging approaches, in particular magnetic resonance imaging (MRI) based techniques provide a powerful means to non-invasively study the effects of physical exercise on human brain structure and function. Employing these techniques in exercise sciences will allow determining central mechanisms of body-brain interactions, both, in health and disease states. It is expected that this may propagate individualized exercise training regimens for disease prevention and, ultimately, adapted applications for prevention and slowing down disease progression in neurodegenerative conditions. At current, however, these imaging techniques are largely based on inter-subject averaging and their impact for personalized medicine with the goal of promoting preventive and personalized healthcare remains to be determined.
\end{abstract}

Keywords Physical exercise $\cdot$ Brain $\cdot$ Magnetic resonance imaging, MRI · Positron emission tomography, PET.

Prevention $\cdot$ Neurodegenerative disease .

Personalized medicine

H. Boecker $(\bowtie)$

Radiologische Universitätsklinik,

FE Klinische Funktionelle Neurobildgebung,

Rheinische Friedrich-Wilhelms-Universität Bonn,

Sigmund-Freud-Str. 25,

53127 Bonn, Germany

e-mail: Henning.Boecker@ukb.uni-bonn.de

\section{Introduction}

Physical exercise has been shown to improve performance in multiple cognitive tasks, including executive control, attentional processing, and spatial memory $[1,2]$. Behavioral findings in humans undergoing regular exercise training have been mainly derived from studies in older cohorts [1-3], but there is growing evidence to support a role of exercise for improved cognitive functioning also in children and young adolescents [4-12].

Exercise-related effects in the central nervous system (CNS) have been attributed to neurogenesis [13-15], angiogenesis $[15,16]$, and synaptic plasticity $[17,18]$. At the molecular level, structural changes appear to be mediated by different neurotrophic factors [19], including brain-derived neurotrophic factor, BDNF [17, 20-22], nerve growth factor, NGF [23], insulin-like growth factor, IGF-1 $[24,25]$, and vascular endothelial growth factor, VEGF [26]. There is evidence from animal studies that exercise is also associated with release of neuronal progenitor cells, which were found to be highly expressed in the surrounding of injured brain tissue [27, 28], and in the hippocampus [29-33]. The hippocampus is known to be a central structure for relational memory acquisition [34-36], and a region decreasing in volume with advanced aging [37, 38]; most notably, atrophy of the hippocampus is considered as a core pathological feature of Alzheimer's Disease (AD) [39-42]. Behavioral data indicate that exercise may alleviate disease progression in $\mathrm{AD}[43,44]$, as well as in other neurodegenerative conditions like Parkinson's Disease [45]. However, the application of in vivo neuroimaging techniques (see below) for monitoring the neuroprotective and restaurative effects of regular exercise in pathologies of the human brain are as yet very limited. 
Given the outlined potential of exercise training for preventive personalized medicine concepts, this review article attempts to highlight the emerging role of modern neuroimaging techniques for monitoring the CNS effects of exercise and, potentially, for providing surrogate markers for clinical trials involving patients and at-risk cohorts. After a brief introduction into the two principal neuroimaging techniques, magnetic resonance imaging (MRI) and positron emission tomography (PET), the current evidence derived from these methods with regard to CNS effects of exercise are summarized. As the so-called "brain activation" studies (with PET or MRI) are mainly considered as research tools requiring across-subject averaging, the emphasis is laid upon imaging structural, metabolic, and neurotransmitter effects of exercise. As there is a quest to apply these methods in longitudinal and counterbalanced protocols, the review also deals with future aspects and applicability of these techniques in health and disease conditions.

\section{Neuroimaging methods}

\section{Magnetic resonance imaging}

MRI can be used to study structural and functional measures in the CNS that are highly relevant in the context of exercise research. The advantage of MRI is its noninvasiveness, allowing repeated measurements in longitudinal study designs. In the following, a brief introduction of MR-based techniques is given for structural gray and white matter imaging, as well as for functional imaging, including MR-spectroscopy, MR perfusion imaging, and functional MRI (fMRI).

\section{Structural imaging: gray matter}

Structural MR imaging provides high-contrast 3D scans with excellent differentiation of cerebrospinal fluid (CBF), gray matter (GM), or white matter (WM). Dedicated sequences allow measuring the entire brain at (sub)millimeter voxel scale. Quantitative structural changes can be determined in cross-sectional designs between different research cohorts (e.g. between low- and high-fit individuals) or in longitudinal designs measuring the effects of long-term interventions (e.g. exercise challenges versus control conditions like stretching).

Quantification of differences in gray matter volume can either be accomplished using classical manual volume-ofinterest (VOI) analyses, semi-automated VOI segmentation algorithms, or automated voxel-based-morphometry (VBM) approaches. VBM quantifies global and/or regional changes in human brain volume, where the probability of voxels being $\mathrm{CBF}, \mathrm{GM}$, or $\mathrm{WM}$ is calculated on the basis of a priori intensity distributions of these tissue types [46]. VBM is an automated technique and as such not prone to observer-bias. However, the interpretation of underlying structural changes is hampered as it is currently impossible to deduce neuronal or vascular changes as primary sources of structural changes over time or between groups, or for instance, whether findings are driven by gyrification differences. A limitation is also that it is impossible to differentiate whether signal changes are due to decreases in gray matter or to increasese in white matter (or vice versa).

In longitudinal studies, tensor-based morphometry (TBM) provides a tool for automated quantification of gray-matter changes over time. In TBM, nonlinear deformation fields are applied to a baseline scan and are subsequently aligned to the follow-up scans, and "based on local analysis of the applied compression and expansion, rates of brain change can be inferred for specific regions of interest or presented in the form of a map" [47].

\section{Structural imaging: white matter}

Directional information of neural tracts connecting brain regions can be determined with another MR technique called diffusion tensor imaging (DTI) [48]. The measured rate of water diffusion will differ depending on the movement direction of water molecules, which is more rapid along anatomical pathways and slower in direction perpendicular to anatomical boundaries. By measuring the direction of this molecular movement in six or more MR gradient directions, it is possible to compute the diffusion tensor (DT). Diffusion anisotropy measures such as the fractional anisotropy (FA) can be derived from the DT, and the direction of the DT can be used to inform about white-matter connectivity (MR-tractography) [49].

\section{Functional MRI}

Increased synaptic activity linked to task performance is associated with local perfusion changes which cause a disproportionate increase of oxygenated hemoglobin in activated brain tissue. The resulting local alteration of the oxy/deoxy-hemoglobin fraction causes a change in netto magnetization of activated brain regions that can be measured with rapid MR-sequences sensitive to the so-called T2* effect [50]. Due to its non-invasiveness (no radiation exposure, no contrast agent exposure), fMRI has become the most influential and most widely distributed functional imaging technique in systems neuroscience. Typically, fMRI is performed either as block design (alternating periods with continuous task performance and control states) or as event-related design (stimuli presented as single events of short duration). 
Based on the observation of default functional activity during resting states which imply intrinsic functional activity of coherent brain networks [51, 52], recent applications of $\mathrm{T} 2 *$-weighted imaging sequences were focused on so-called "resting brain" studies [53-58], in which functional connectivity is determined during rest states. Functional connectivity is assumed when coherent spontaneous signal time-courses are found over time in distant regions of the brain.

\section{Perfusion MRI}

It is nowadays possible to determine brain perfusion, both globally and locally, with MR-based arterial spin labeling (ASL) [59, 60] which is now also applied in clinical populations [61]. ASL is a non-invasive technique and, in contrast to $\mathrm{H}_{2}{ }^{15} \mathrm{O}$ PET, global and local perfusion can be quantified without any radiation exposure. ASL is based on the magnetic labeling of the inflowing water in the carotid arteries. The technique is relatively new and direct comparative studies with PET, the current gold standard in perfusion mapping, are as yet limited [62]. Thus, the validity of CASL, in particular for longitudinal intervention studies will need to be further determined in direct comparison to PET. Figure 1 outlines the method.

\section{MR-Spectroscopy}

Proton magnetic resonance spectroscopy (1H-MRS) is a non-invasive technique that allows quantification of brain metabolites, either as localized single voxel spectroscopy or as 2D or 3D spectroscopic imaging. Various brain metabolites (e.g. N-acetylaspartate, choline compounds, (phospho)creatine, myo-inositol, lactate) can be quantified yielding their absolute concentrations or metabolite ratios, and changes related to exercise interventions can potentially be identified either cross-sectionally, or in longitudinal designs. Regarding the postulated neuroprotective and restaurative effects of exercise, quantification of $\mathrm{N}$-acetylaspartate levels may be particularly interesting as this compound is considered to represent a neuronal marker and a measure of neuronal integrity $[63,64]$. Figure 2 depicts a proton spectrum at $3 \mathrm{~T}$ from the hippocampus volume depicted in Fig. 3.

\section{Positron emission tomography}

PET is a technique based on annihilation coincidence detection [65]. The production of suitably positronemitters depends on a local cyclotron unit and a radiochemistry laboratory in nearby distance to a PET scanner, at least when radionuclides with short half-lifes are employed. Only radiocompounds with longer half-lifes, for instance 18-F-labeled compounds (half-life $109.8 \mathrm{~min}$ ), can be transported over longer distances. Due to the inherent radiation exposure of PET, this technique has limited applicability, in particular in studies that require repeated measurements over time.

Using specific radioligands, absolute quantification of receptor binding is possible in vivo and PET may thus be highly informative about the central neurochemistry of exercise, in particular opioidergic (and endocannabinoid), dopaminergic, and serotoninergic transmission. Neuroreceptor PET may also impact on our understanding of central neurochemical mechanisms of exercise in different disease states, for instance affective disorders, which are
Fig. 1 Quantitative perfusion map using CASL in single subject (single-shot spin-echo echoplanar imaging at $3.0 \mathrm{~T}, \mathrm{TR} / \mathrm{TE}$ 4200/38, 11slices, Slice thickness: $8 \mathrm{~mm}$, Matrix: $64 \times 64$, "labeling delay" $700 \mathrm{~ms}, 40$ dynamics, scan-time: 336 s.,Transmit/Receive Head Coil). Courtesy Dr. Lukas Scheef, Dept. of Radiology, University Bonn

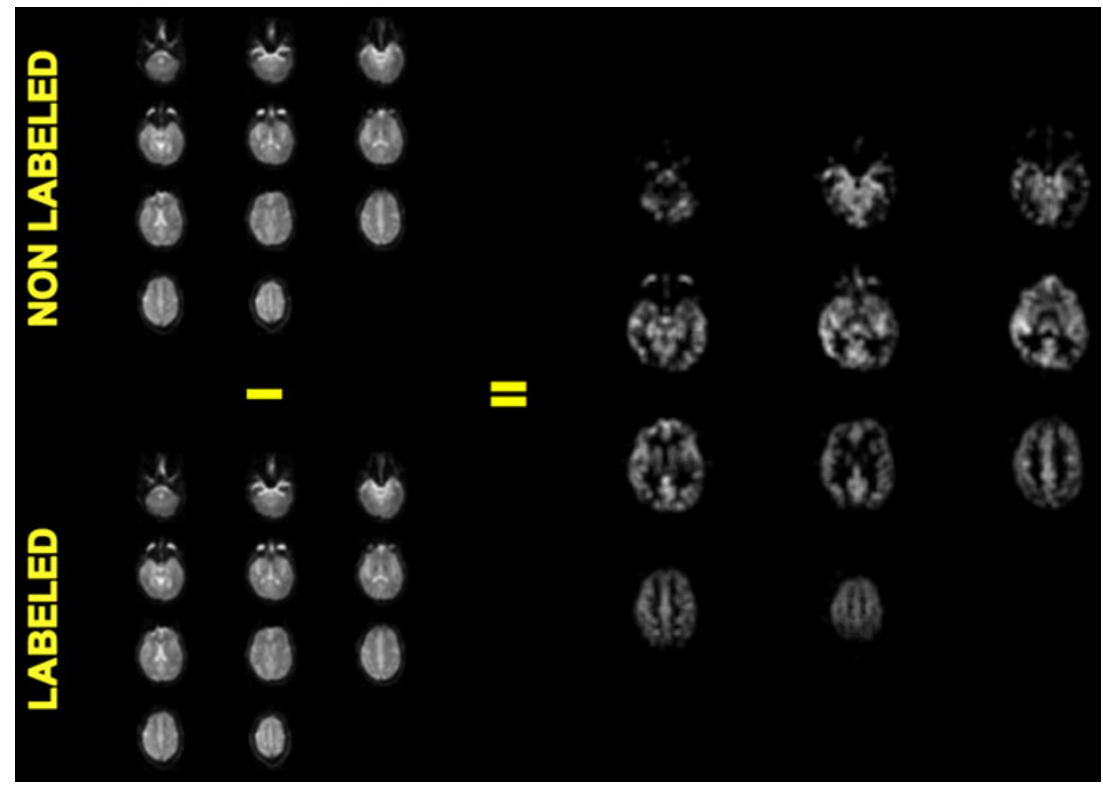



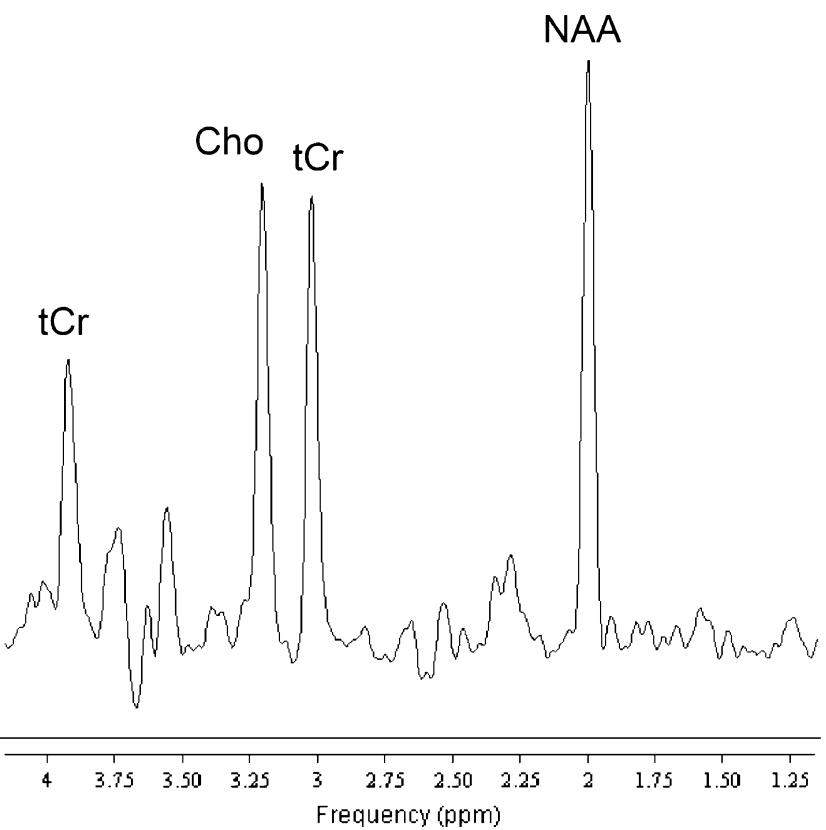

Fig. 2 Water-suppressed single-voxel spectrum with TR/TE 2000/140 ms from a $6.2 \mathrm{ccm}$-VOI centered on the left hippocampus of a healthy control (male/37y). Bandwidth $2 \mathrm{kHz}$ for 1024 data points, 128 signal averages yielding an acquisition time of 4:20 min (courtesy Dr. Frank Träber, Dept. of Radiology, University Bonn)

known to benefit substantially from regular exercise regimens [66, 67].

The tracer ${ }^{18}$ F-Fluoro-deoxy-glucose (FDG) is well established for quantification of brain metabolism, both regionally and globally, and this measure will be informative in exercise applications in neurodegenerative disease conditions. Eventually, using novel compounds [68, 69], PET might be applied for in vivo monitoring of distinct pathological measures, for instance clearance of amyloid places with exercise in subjects with amyloid-related pathologies.

\section{Neuroimaging research in exercise science}

There is clear evidence that the brain undergoes substantial structural and metabolic changes with increasing age, which is associated with declines in cognitive performance. Age-associated regional brain atrophy is most significant in the prefrontal cortex $[70,71]$ and in the medial temporal lobe including the entorhinal and hippocampal regions [72], while other brain regions like sensorimotor and occipital cortex remain relatively spared. In accord with the animal literature (see above) there are now also indications from human imaging studies to support the notion that exercise alters this age-related decline. In the following part of this review, current data on structural and functional effects of various exercise challenges in different age groups will be summarized.

Structural brain changes induced by exercise

Based on a cross-sectional MR-study design, Colcombe et al. were the first to report a positive association between brain tissue density and aerobic fitness levels [73]. They acquired high-resolution structural MRI data and performed VBM analyses in a population of 55 older adults (55-79 year) with estimated VO2 scores (derived from the Rockport 1-mile walk protocol) ranging from 11-50. They described declines in gray matter tissue densities in prefrontal, superior parietal, and middle/inferior temporal cortices as a function of increasing age [73]. Interestingly, volume losses in these identical areas (i.e. affected by age)

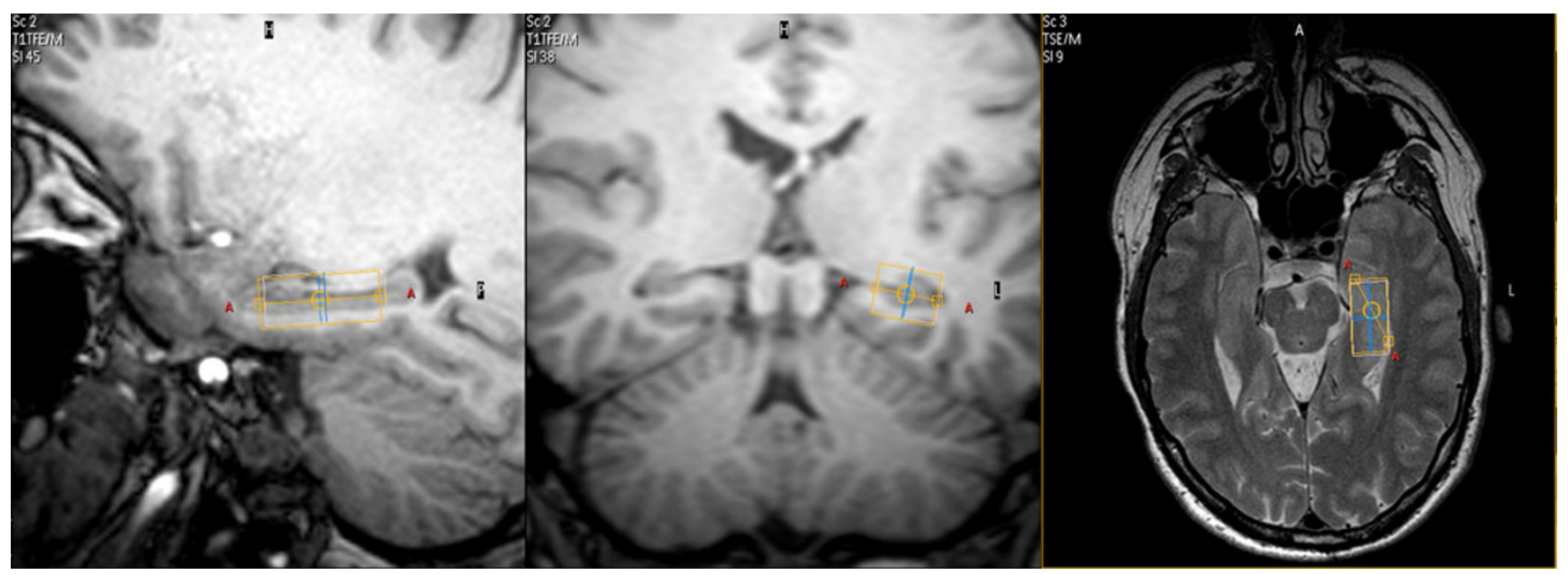

Fig. 3 Delineation of a VOI centered on the left hippocampus of a healthy control for single-voxel spectroscopy (see Fig. 3; courtesy Dr. Lukas Scheef, Dept. of Radiology, University Bonn) 
were substantially reduced as a function of cardiovascular fitness. Although on a cross-sectional level, these authors provided first human evidence for a reciprocal link between increasing cardiovascular fitness and decreasing neural degeneration in elderly subjects. Interestingly, grey matter density changes depending on aerobic capacity levels have been reported also for the anterior insula, an area implicated in cardiovascular control during exercise and implicated in autonomic arousal: Using VBM analysis in a sample of 33 healthy young subjects (mean age 23.9, range 19-35 year), Peters et al. demonstrated that aerobic capacity was positively associated with structural changes in right anterior insula [74]. More recent cross-sectional morphometry work extended these findings by explicitly focusing on temporal lobe structures, i.e. where animal work has found structural (mainly hippocampal) plasticity following regular physical exercise $[13,18]$ : Bugg et al. used an automated labeling technique of the mesial temporal lobe, and found in 52 older adults aged 55-79 year that this particular region undergoes a steady age-related decline in low-fit individuals, whereas the age-related decline is attenuated in high-fit individuals [75]. Substantial differences in hippocampal size depending on fitness levels were also found in childen at the age of 9-10 year [76], but it has to be pointed out that any causative interpretations derived from cross-sectional study designs have to be considered with caution.

Longitudinal intervention trials are methodologically more stringent and studies from Arthur Kramer's group at the Beckmann Institute, Urbana, Illinois, have shown the path to be pursued in future structural imaging studies of exercise: In 2006, Colcombe et al. published the first randomized clinical morphometry trial investigating the effects of physical exercise on structural brain changes [77]. They included 59 sedentary volunteers (60-79 years) in a 6-month randomized clinical trial, in which half of the cohort was allocated to aerobic training, the other half to toning and stretching [77]. Gray matter increases were identified in the exercise group, but not in the control group, and occurred in frontal lobe structures involved in higher order attentional control and memory (e.g. dorsal anterior cingulate cortex, supplementary motor area, middle frontal gyrus bilaterally, right inferior frontal gyrus). Differential effects induced by exercise training have also been reported in younger adults (control group: 21.2 \pm 1.5 year, training group: $20.9 \pm 3.2$ year), as evidenced by a significant group $\mathrm{x}$ time interaction in the left anterior insula, which was mainly driven by a volume decrease in the control group over approximately 4 months [78].

Most recently, Erickson et al. performed an intervention trial involving 160 sedentary subjects, which were randomized to either low-intensity exercise or stretching [79]. They applied an automated labeling (ROI) analysis and found an increase of hippocampal volume by $1-2 \%$ over a time- period of 12 months in the exercise group, whereas decreases in hippocampal volume of the same magnitude were observed in the control group (i.e. representing age-related declines). Similar effects were observed for the left and the right hippocampus, but no significant changes in size were observed in the caudate nucleus or the thalamus. Interestingly, the hippocampal volume increases in the exercise group were correlated with improved performance on a spatial memory task, thus, revealing a link between structure and function in a region-specific manner. This longitudinal study with repeated MRI measures taken at six and at 12 months highlights the dynamics of structural plasticity changes at the hippocampal level induced by exercise.

To conclude, recent randomized trials in healthy sedentary cohorts (with either exercise or low level control conditions) substantiate the morphometry data from earlier cross-sectional studies. They confirm that physical exercise is a powerful modulator of cortical and subcortical brain structure. Another interesting structural parameter in this context is "cortical thickness", which can be automatically measured across the cortical mantle. For instance, mental memory training has been shown to induce regional increases in cortical thickness, with thickness changes in the right fusiform and lateral orbitofrontal cortex correlating positively with improvement in source memory performance [80].

Thus far, no longitudinal randomized studies have examined the effects of exercise on brain structure in patient cohorts. A cross-sectional study in patients with $\mathrm{AD}$, however, showed with VBM that there is a inverse relationship between fitness levels and the amount of hippocampal atrophy [81]. Another study examined the association of cardiorespiratory fitness and global brain atrophy and cognition in early-stage AD and found reduced global brain atrophy in patients with increased cardiorespiratory fitness [44]. Hence, cardiorespiratory fitness may moderate brain atrophy in neurodegenerative disorders like AD but, clearly, future studies in randomized longitudinal intervention trials need to be conducted in these patients and also extending to at-risk cohorts.

Functional brain changes induced by exercise

As mentioned in the introduction, this review will not go into depth regarding so-called brain activation studies applied in exercise sciences because functional task and resting brain studies are considered above all as research tools requiring accross-subject averaging procedures. It is expected that this will presumably also hamper their applicability as predictive, preventive and personalized surrogate markers in future, as task processing is considerably affected by interindividual variance. Nevertheless, it is important to point out that the effects of exercise training on 
brain-related activity during cognitive task performance have provided some interesting findings that are compatible with a modulatory effect of exercise on task-specific cognitive processing.

A focus has been laid on the effects of exercise on attentional control mechanisms, again pioneered by Arthur Kramer's group, using a modified flanker task, in which subjects have to focus on the direction of a center arrow (leftward/rightward) while ignoring four surrounding flanker arrows, either pointing in the same (congruent) or the opposite (incongruent) direction. This task requires continued attentional control and suppression of irrelevant information. In a cross-sectional design, brain activity reflecting the difference between processing inconsistent versus consistent conditions was related to cardiorespiratory fitness levels of the participats. Higher fit older adults showed relatively increased activation in the dorsolateral prefrontal cortex on the right side and relatively reduced activity in the anterior cingulate cortex, implicating that improved performance in the high fit group can be explained by improved top-down control and reduced experience of conflict [82].

When the same task was tested under longitudinal conditions in 29 subjects (mean 65.60 year, range 58-77 year old), before and after either six-month moderate intensity walking (intervention group) or stretching and toning (control group), the results could be further substantiated [82]: Exercise increased fitness and, consistent with the results from the cross-sectional study, exercise increased activity in the DLPFC and decreased activity in the ACC. As these effects were only seen in the exercise group, the interpretation is that aerobic exercise is a specific modulator of cognitve processing.

As exercise effects are discussed in the context of prophylactic influences of cardiorespiratory fitness on cognitive decline in older adults (see also section on structural imaging), future activation studies will have to pursue the important question whether fitness also moderates disease-related abnormal cognitive processing in neurodegenerative conditions and whether restaurative effects on brain function can be found in longitudinal functional imaging designs.

Metabolic brain changes and neurotransmission induced by exercise

So far, PET with the tracer ${ }^{18} \mathrm{~F}-\mathrm{FDG}$ has been performed only to study the effects of acute exercise bouts, as shown in a paradigm where subjects were instructed to run $10-15 \mathrm{~min}$ before and subsequently continue running for a total of 30-40 min after ${ }^{18} \mathrm{~F}-\mathrm{FDG}$ injection [83]. Upon imaging, acute exercise - as compared with the resting state - was associated with an increased metabolic rate of glucose consumption in various cortical areas, including premotor and sensori-motor areas, as well as in the cerebellum. These intial applications highlight the potential of ${ }^{18} \mathrm{~F}$-FDG PET in exercise neuroscience; indeed, rather than using the method for examining acute exercise bouts, ${ }^{18}$ F-FDG PET may have an even greater potential for monitoring sustained metabolic effects of regular physical exercise and, thereby, to provide in vivo signatures of neuroprotective effects and/or restauration of brain metabolism due to chronic exercise training. While human studies of this kind have not yet been published, animal research has demonstated increases in metabolic capacity after repeated voluntary running wheel exercise in rats, i.e. indexed as increases in cytochromoxydase activity [84]. ${ }^{18}$ F-FDG PET is at present considered as a "gold standard" method for detection of metabolic changes in patients with neurodegenerative disorders. $\mathrm{AD}$ is characterized by a specific pattern of regional metabolic dysfunction and this pattern can be demonstrated already in early presymptomatic stages of disease with high sensitivity and specificity. Thus, ${ }^{18} \mathrm{~F}-\mathrm{FDG}$ PET would be ideally suited in combination with neuropsychological testing to quantify metabolic adaptations to exercise in $\mathrm{AD}$ patients and at-risk subjects.

Furthermore, as PET also allows more specific in vivo characterization of $\mathrm{AD}$ pathophysiology using tracers targeting amyloid plaques [68, 69], it is also foreseeable that these so-called "amyloid tracers" will be used to measure amyloid clearance associated with exercise in preclinical stages of AD. These and other future applications are unique, as pathophysiological substrates of disease pathologies can be directly monitored in randomized clinical trials and, hopefully, will foster applicability of moderate exercise interventions in disease conditions for prevention and/or restauration.

PET ligand studies have also been applied in exercise research to study acute release of specific neurotransmitters in vivo. The measurement of acute neurotransmission induced by acute exercise bouts has so far been limited to the dopaminergic [85] and opioidergic [86] systems, which are highly relevant for reward processing and mood in exercise. It is, however, also well conceivable that PET will be highly informative in studies focusing on long-term effects of exercise on neurotransmission and receptor distribution. Thereby, PET may generate new understanding of underlying central neurochemical mechanisms of exercise (in particular opioidergic, endocannabinoid, dopaminergic, and serotinergic transmission) and how this impacts on affective disease states. Indeed, patients with affective disorders are known to benefit from regular exercise regimens [66, 67], hence, studies on long-term adaptations of neurotransmitter systems following regular exercise will be of particular interest in future studies, both, in treatment and prevention settings. 


\section{Conclusions \& outlook}

Although the application of modern neuroimaging techniques in human exercise research is still in its infancy, there are indications that regular exercise promotes the structural and functional integrity of the CNS and, thereby, counteracts agerelated decline. The rationale behind exercise interventions goes beyond direct regenerative effects of exercise (neurogenesis, angiogenesis, synaptic plasticity), as exercise effects are also driven by a reduction of cardiovascular risk profiles and associated morbidity.

In terms of preventive and predictive medicine, in vivo biomarkers are needed in the future that allow determining functional or structural brain changes in single individuals with a high degree of sensitivity and specificity. However, subtle changes are difficult to detect with imaging and current research studies are usually done in larger cohorts to allow statistical inference and - by applying corrections for multiple comparisons - valuable generalization to larger populations. Thus, not all directions pursued in research are directly applicable as valuable imaging tools suitable for single subject studies. This is particularly true for the field of activation studies, whereas metabolic effects may be reliably monitored in single subjects, depending on the robustness of the applied methods.

In conclusion, the structural and functional outcome measures described in this review article can not yet be considered as individual surrogate markers in the context of personalized physical exercise recommendations. This has to do with the fact, that the imaging data described here subserve mostly research purposes, and, likewise are derived from larger cohorts and use across-subject averaging. Nevertheless, in vivo whole-brain neuroimaging studies with PET and MRI derived from research cohorts will permit identifying outcome measures of physical exercise treatments and, thereby, inform researchers about underlying structural, metabolic, neuronal and potentially neurotransmitter effects of exercise. This will impact upon personalized medicine and provide strong grounds for exercise applications in healthy subjects, and even more so in disease states and preclinical situations. Thus, application of neuroimaging in exercise research will be a valuable human research tool that will close the gap between animal research at the one hand and pure behavioral studies on the other hand. At least for structural and metabolic imaging, applicability in personalized settings using non-invasive imaging in longitudinal settings is not totally inconceivable, however, the future applicability in predictive, preventive, and personalized medicine depends on the sensitivity of neuroimaging to detect exercise-interventions on a single-subject basis. As yet, the effect sizes are still largely unknown and further research in cohort studies is necessary to gain further insight into the modulatory effects of exercise on the human brain.

\section{References}

1. Kramer AF, Colcombe SJ, McAuley E, Eriksen KI, Scalf P, Jerome GJ, et al. Enhancing brain and cognitive function of older adults through fitness training. J Mol Neurosci. 2003;20(3):213-21.

2. Colcombe S, Kramer AF. Fitness effects on the cognitive function of older adults: a meta-analytic study. Psychol Sci. 2003;14 (2):125-30.

3. Erickson KI, Kramer AF. Aerobic exercise effects on cognitive and neural plasticity in older adults. Br J Sports Med. 2009;43 (1):22-4.

4. Chaddock L, Hillman CH, Buck SM, Cohen NJ. Aerobic fitness and executive control of relational memory in preadolescent children. Med Sci Sports Exerc. 2011;43(2):344-9.

5. Wu CT, Pontifex MB, Raine LB, Chaddock L, Voss MW, Kramer $\mathrm{AF}$, et al. Aerobic fitness and response variability in preadolescent children performing a cognitive control task. Neuropsychology 2011; 25:333-41.

6. Themanson JR, Pontifex MB, Hillman CH. Fitness and action monitoring: evidence for improved cognitive flexibility in young adults. Neuroscience. 2008;157(2):319-28.

7. Pontifex MB, Raine LB, Johnson CR, Chaddock L, Voss MW, Cohen NJ, et al. Cardiorespiratory fitness and the flexible modulation of cognitive control in preadolescent children. J Cogn Neurosci. 2011;23(6):1332-45.

8. Hillman $\mathrm{CH}$, Erickson KI, Kramer AF. Be smart, exercise your heart: exercise effects on brain and cognition. Nat Rev Neurosci. 2008;9(1):58-65.

9. Hillman CH, Kamijo K, Scudder M. A review of chronic and acute physical activity participation on neuroelectric measures of brain health and cognition during childhood. Prev Med. 2011; 52 Suppl 1: S21-8.

10. Hillman CH, Motl RW, Pontifex MB, Posthuma D, Stubbe JH, Boomsma DI, et al. Physical activity and cognitive function in a cross-section of younger and older community-dwelling individuals. Health Psychol. 2006;25(6):678-87.

11. Hillman CH, Buck SM, Themanson JR, Pontifex MB, Castelli DM. Aerobic fitness and cognitive development: Event-related brain potential and task performance indices of executive control in preadolescent children. Dev Psychol. 2009;45(1):114-29.

12. Hillman $\mathrm{CH}$, Castelli DM, Buck SM. Aerobic fitness and neurocognitive function in healthy preadolescent children. Med Sci Sports Exerc. 2005;37(11):1967-74.

13. van Praag H, Kempermann G, Gage FH. Running increases cell proliferation and neurogenesis in the adult mouse dentate gyrus. Nat Neurosci. 1999;2(3):266-70.

14. van Praag H, Shubert T, Zhao C, Gage FH. Exercise enhances learning and hippocampal neurogenesis in aged mice. J Neurosci. 2005;25(38):8680-5.

15. Van der Borght K, Kobor-Nyakas DE, Klauke K, Eggen BJ, Nyakas C, Van der Zee EA, et al. Physical exercise leads to rapid adaptations in hippocampal vasculature: temporal dynamics and relationship to cell proliferation and neurogenesis. Hippocampus. 2009;19(10):928-36.

16. Ding YH, Li J, Zhou Y, Rafols JA, Clark JC, Ding Y. Cerebral angiogenesis and expression of angiogenic factors in aging rats after exercise. Curr Neurovasc Res. 2006;3(1):15-23.

17. Gomez-Pinilla F, Ying Z, Roy RR, Molteni R, Edgerton VR. Voluntary exercise induces a BDNF-mediated mechanism that promotes neuroplasticity. J Neurophysiol. 2002;88(5):2187-95.

18. Cotman CW, Berchtold NC. Exercise: a behavioral intervention to enhance brain health and plasticity. Trends Neurosci. 2002;25 (6):295-301.

19. Cotman CW, Berchtold NC, Christie LA. Exercise builds brain health: key roles of growth factor cascades and inflammation. Trends Neurosci. 2007;30(9):464-72. 
20. Gomez-Pinilla F, Vaynman S, Ying Z. Brain-derived neurotrophic factor functions as a metabotrophin to mediate the effects of exercise on cognition. Eur J Neurosci. 2008;28(11):2278-87.

21. Griesbach GS, Hovda DA, Gomez-Pinilla F. Exercise-induced improvement in cognitive performance after traumatic brain injury in rats is dependent on BDNF activation. Brain Res. 2009;1288:10515.

22. Vaynman S, Ying Z, Gomez-Pinilla F. Hippocampal BDNF mediates the efficacy of exercise on synaptic plasticity and cognition. Eur J Neurosci. 2004;20(10):2580-90.

23. Ang ET, Gomez-Pinilla F. Potential therapeutic effects of exercise to the brain. Curr Med Chem. 2007;14(24):2564-71.

24. Lopez-Lopez C, LeRoith D, Torres-Aleman I. Insulin-like growth factor $\mathrm{I}$ is required for vessel remodeling in the adult brain. Proc Natl Acad Sci USA. 2004;101(26):9833-8.

25. Llorens-Martin M, Torres-Aleman I, Trejo JL. Mechanisms mediating brain plasticity: IGF1 and adult hippocampal neurogenesis. Neuroscientist. 2009;15(2):134-48.

26. Fabel K, Tam B, Kaufer D, Baiker A, Simmons N, Kuo CJ, et al. VEGF is necessary for exercise-induced adult hippocampal neurogenesis. Eur J Neurosci. 2003;18(10):2803-12.

27. Itoh T, Imano M, Nishida S, Tsubaki M, Hashimoto S, Ito A, et al. Exercise increases neural stem cell proliferation surrounding the area of damage following rat traumatic brain injury. J Neural Transm. 2011;118(2):193-202.

28. Jin J, Kang HM, Park C. Voluntary exercise enhances survival and migration of neural progenitor cells after intracerebral haemorrhage in mice. Brain Inj. 2010;24(3):533-40.

29. Wu CW, Chang YT, Yu L, Chen HI, Jen CJ, Wu SY, et al. Exercise enhances the proliferation of neural stem cells and neurite growth and survival of neuronal progenitor cells in dentate gyrus of middle-aged mice. J Appl Physiol. 2008;105(5):1585-94.

30. Brandt MD, Maass A, Kempermann G, Storch A. Physical exercise increases Notch activity, proliferation and cell cycle exit of type-3 progenitor cells in adult hippocampal neurogenesis. Eur J Neurosci. 2010;32(8):1256-64.

31. Kempermann G, van Praag H, Gage FH. Activity-dependent regulation of neuronal plasticity and self repair. Prog Brain Res. 2000;127:35-48.

32. Kim YP, Kim H, Shin MS, Chang HK, Jang MH, Shin MC, et al. Age-dependence of the effect of treadmill exercise on cell proliferation in the dentate gyrus of rats. Neurosci Lett. 2004;355 (1-2):152-4.

33. Lou SJ, Liu JY, Chang H, Chen PJ. Hippocampal neurogenesis and gene expression depend on exercise intensity in juvenile rats. Brain Res. 2008;1210:48-55.

34. Cohen NJ, Ryan J, Hunt C, Romine L, Wszalek T, Nash C. Hippocampal system and declarative (relational) memory: summarizing the data from functional neuroimaging studies. Hippocampus. 1999;9(1):83-98.

35. Davachi L. Item, context and relational episodic encoding in humans. Curr Opin Neurobiol. 2006;16(6):693-700.

36. Squire LR. Memory and the hippocampus: a synthesis from findings with rats, monkeys, and humans. Psychol Rev. 1992;99 (2):195-231.

37. Golomb J, de Leon MJ, Kluger A, George AE, Tarshish C, Ferris $\mathrm{SH}$. Hippocampal atrophy in normal aging. An association with recent memory impairment. Arch Neurol. 1993;50(9):967-73.

38. Golomb J, Kluger A, de Leon MJ, Ferris SH, Mittelman M, Cohen $\mathrm{J}$, et al. Hippocampal formation size predicts declining memory performance in normal aging. Neurology. 1996;47 (3):810-3.

39. Barnes J, Bartlett JW, van de Pol LA, Loy CT, Scahill RI, Frost C, et al. A meta-analysis of hippocampal atrophy rates in Alzheimer's disease. Neurobiol Aging. 2009;30(11):1711-23.
40. de Toledo-Morrell L, Stoub TR, Wang C. Hippocampal atrophy and disconnection in incipient and mild Alzheimer's disease. Prog Brain Res. 2007;163:741-53.

41. Chetelat G, Baron JC. Early diagnosis of Alzheimer's disease: contribution of structural neuroimaging. Neuroimage. 2003;18 (2):525-41.

42. de Leon MJ, Convit A, George AE, Golomb J, de Santi S, Tarshish C, et al. In vivo structural studies of the hippocampus in normal aging and in incipient Alzheimer's disease. Ann N Y Acad Sci. 1996;777:1-13.

43. Archer T. Physical exercise alleviates debilities of normal aging and Alzheimer's disease. Acta Neurol Scand. 2011;123(4):22138.

44. Burns JM, Cronk BB, Anderson HS, Donnelly JE, Thomas GP, Harsha A, et al. Cardiorespiratory fitness and brain atrophy in early Alzheimer disease. Neurology. 2008;71(3):210-6.

45. Hirsch MA, Farley BG. Exercise and neuroplasticity in persons living with Parkinson's disease. Eur J Phys Rehabil Med. 2009;45 (2):215-29.

46. Ashburner J, Friston KJ. Voxel-based morphometry-the methods. Neuroimage. 2000;11(6 Pt 1):805-21.

47. Leow AD, Klunder AD, Jack Jr CR, Toga AW, Dale AM, Bernstein MA, et al. Longitudinal stability of MRI for mapping brain change using tensor-based morphometry. Neuroimage. 2006;31(2):627-40.

48. Moseley ME, Cohen Y, Kucharczyk J, Mintorovitch J, Asgari HS, Wendland MF, et al. Diffusion-weighted MR imaging of anisotropic water diffusion in cat central nervous system. Radiology. 1990;176 (2):439-45.

49. Conturo TE, Lori NF, Cull TS, Akbudak E, Snyder AZ, Shimony JS, et al. Tracking neuronal fiber pathways in the living human brain. Proc Natl Acad Sci USA. 1999;96(18):10422-7.

50. Ogawa S, Lee TM, Kay AR, Tank DW. Brain magnetic resonance imaging with contrast dependent on blood oxygenation. Proc Natl Acad Sci USA. 1990;87(24):9868-72.

51. Raichle ME, MacLeod AM, Snyder AZ, Powers WJ, Gusnard DA, Shulman GL. A default mode of brain function. Proc Natl Acad Sci USA. 2001;98(2):676-82.

52. Raichle ME, Snyder AZ. A default mode of brain function: a brief history of an evolving idea. Neuroimage. 2007;37(4):1083-90. discussion 1097-9.

53. Fox MD, Greicius M. Clinical applications of resting state functional connectivity. Front Syst Neurosci. 2010;4:19.

54. Greicius MD, Supekar K, Menon V, Dougherty RF. Resting-state functional connectivity reflects structural connectivity in the default mode network. Cereb Cortex. 2009;19(1):72-8.

55. De Luca M, Beckmann CF, De Stefano N, Matthews PM, Smith SM. fMRI resting state networks define distinct modes of long-distance interactions in the human brain. Neuroimage. 2006;29(4):135967.

56. Damoiseaux JS, Rombouts SA, Barkhof F, Scheltens P, Stam CJ, Smith SM, et al. Consistent resting-state networks across healthy subjects. Proc Natl Acad Sci USA. 2006;103(37):13848-53.

57. De Luca M, Smith S, De Stefano N, Federico A, Matthews PM. Blood oxygenation level dependent contrast resting state networks are relevant to functional activity in the neocortical sensorimotor system. Exp Brain Res. 2005;167(4):587-94.

58. Fransson P. Spontaneous low-frequency BOLD signal fluctuations: an fMRI investigation of the resting-state default mode of brain function hypothesis. Hum Brain Mapp. 2005;26(1):15-29.

59. Williams DS, Detre JA, Leigh JS, Koretsky AP. Magnetic resonance imaging of perfusion using spin inversion of arterial water. Proc Natl Acad Sci USA. 1992;89(1):212-6.

60. Detre JA, Leigh JS, Williams DS, Koretsky AP. Perfusion imaging. Magn Reson Med. 1992;23(1):37-45. 
61. Scheef L, Manka C, Daamen M, Kuhn KU, Maier W, Schild HH, et al. Resting-state perfusion in nonmedicated schizophrenic patients: a continuous arterial spin-labeling 3.0-T MR study. Radiology. 2010;256(1):253-60.

62. Kimura H, Kado H, Koshimoto Y, Tsuchida T, Yonekura Y, Itoh H. Multislice continuous arterial spin-labeled perfusion MRI in patients with chronic occlusive cerebrovascular disease: a correlative study with CO2 PET validation. J Magn Reson Imaging. 2005;22 (2):189-98.

63. Kantarci K. 1H magnetic resonance spectroscopy in dementia. $\mathrm{Br}$ J Radiol. 2007 Dec;80 Spec No 2:S146-52.

64. Moffett JR, Ross B, Arun P, Madhavarao CN, Namboodiri AM. $\mathrm{N}$-Acetylaspartate in the CNS: from neurodiagnostics to neurobiology. Prog Neurobiol. 2007;81(2):89-131.

65. Phelps ME, Hoffman EJ, Mullani NA, Ter-Pogossian MM. Application of annihilation coincidence detection to transaxial reconstruction tomography. J Nucl Med. 1975;16(3):210-24.

66. Canbeyli R. Sensorimotor modulation of mood and depression: an integrative review. Behav Brain Res. 2010;207(2):249-64.

67. Guszkowska M. Effects of exercise on anxiety, depression and mood. Psychiatr Pol. 2004;38(4):611-20.

68. Mathis CA, Lopresti BJ, Klunk WE. Impact of amyloid imaging on drug development in Alzheimer's disease. Nucl Med Biol. 2007;34(7):809-22.

69. Klunk WE, Engler H, Nordberg A, Wang Y, Blomqvist G, Holt DP, et al. Imaging brain amyloid in Alzheimer's disease with Pittsburgh Compound-B. Ann Neurol. 2004;55(3):306-19.

70. Lemaitre H, Goldman AL, Sambataro F, Verchinski BA, Meyer-Lindenberg A, Weinberger DR, et al. Normal age-related brain morphometric changes: nonuniformity across cortical thickness, surface area and gray matter volume? Neurobiol Aging. 2010. doi:10.1016/j.neurobiolaging.2010.07.013

71. Resnick SM, Pham DL, Kraut MA, Zonderman AB, Davatzikos C. Longitudinal magnetic resonance imaging studies of older adults: a shrinking brain. J Neurosci. 2003;23(8):3295-301.

72. Raz N, Lindenberger U, Rodrigue KM, Kennedy KM, Head D, Williamson A, et al. Regional brain changes in aging healthy adults: general trends, individual differences and modifiers. Cereb Cortex. 2005;15(11):1676-89.

73. Colcombe SJ, Erickson KI, Raz N, Webb AG, Cohen NJ, McAuley $\mathrm{E}$, et al. Aerobic fitness reduces brain tissue loss in aging humans. J Gerontol A Biol Sci Med Sci. 2003;58(2):176-80.
74. Peters J, Dauvermann M, Mette C, Platen P, Franke J, Hinrichs T, et al. Voxel-based morphometry reveals an association between aerobic capacity and grey matter density in the right anterior insula. Neuroscience. 2009;163(4):1102-8.

75. Bugg JM, Head D. Exercise moderates age-related atrophy of the medial temporal lobe. Neurobiol Aging. 2011;32(3):506-14.

76. Chaddock L, Erickson KI, Prakash RS, Kim JS, Voss MW, Vanpatter $\mathrm{M}$, et al. A neuroimaging investigation of the association between aerobic fitness, hippocampal volume, and memory performance in preadolescent children. Brain Res. 2010;1358:172-83.

77. Colcombe SJ, Erickson KI, Scalf PE, Kim JS, Prakash R, McAuley $\mathrm{E}$, et al. Aerobic exercise training increases brain volume in aging humans. J Gerontol A Biol Sci Med Sci. 2006;61(11):1166-70.

78. Gondoh Y, Sensui H, Kinomura S, Fukuda H, Fujimoto T, Masud $\mathrm{M}$, et al. Effects of aerobic exercise training on brain structure and psychological well-being in young adults. J Sports Med Phys Fitness. 2009;49(2):129-35.

79. Erickson KI, Voss MW, Prakash RS, Basak C, Szabo A, Chaddock $\mathrm{L}$, et al. Exercise training increases size of hippocampus and improves memory. Proc Natl Acad Sci USA. 2011;108(7):3017-22.

80. Engvig A, Fjell AM, Westlye LT, Moberget T, Sundseth O, Larsen VA, et al. Effects of memory training on cortical thickness in the elderly. Neuroimage. 2010;52(4):1667-76.

81. Honea RA, Thomas GP, Harsha A, Anderson HS, Donnelly JE, Brooks WM, et al. Cardiorespiratory fitness and preserved medial temporal lobe volume in Alzheimer disease. Alzheimer Dis Assoc Disord. 2009;23(3):188-97.

82. Colcombe SJ, Kramer AF, Erickson KI, Scalf P, McAuley E, Cohen NJ, et al. Cardiovascular fitness, cortical plasticity, and aging. Proc Natl Acad Sci USA. 2004;101(9):3316-21.

83. Tashiro M, Itoh M, Fujimoto T, Masud MM, Watanuki S, Yanai K. Application of positron emission tomography to neuroimaging in sports sciences. Methods. 2008;45(4):300-6.

84. McCloskey DP, Adamo DS, Anderson BJ. Exercise increases metabolic capacity in the motor cortex and striatum, but not in the hippocampus. Brain Res. 2001;891(1-2):168-75.

85. Wang GJ, Volkow ND, Fowler JS, Franceschi D, Logan J, Pappas NR, et al. PET studies of the effects of aerobic exercise on human striatal dopamine release. J Nucl Med. 2000;41(8):1352-6.

86. Boecker H, Sprenger T, Spilker ME, Henriksen G, Koppenhoefer $\mathrm{M}$, Wagner KJ, et al. The runner's high: opioidergic mechanisms in the human brain. Cereb Cortex. 2008;18(11):2523-31. 